\title{
Semantic Wikis as Platforms for Teaching and Learning Support in Higher Education
}

\author{
Lamia Berkani \\ Dept. of Computer Science, USTHB
}

\begin{abstract}
This paper addresses the need to support learning and teaching in higher education context. We consider the collaboration between instructors and between learners through online communities. We focus on semantic wikis as platforms allowing innovative means for creating and sharing knowledge between members of those communities. We propose an Adaptive Semantic Wiki called EasyLearningWiki, based on the semantic web technologies in order to enhance the knowledge sharing and reuse, offering the functionalities of a wiki together with some knowledge management features. EasyLearningWiki is based on an ontology used to describe the learning resources through the objective annotations and to express the member's feedback through the subjective annotations. Furthermore, we describe the member's profile in order to allow an adaptive access to the semantic wiki.
\end{abstract}

\section{Introduction}

Educational systems around the world are under increasing pressure to improve quality, increase efficiency, and use the new information and communication technologies to teach students the knowledge and skills they need in the $21_{\text {st }}$ century. Improving the quality of education through the diversification of methods, innovation, sharing, communication, and best practices are one of the most important strategies for managing the educational system process.

The use of technology in education, commonly defined as e-learning, has become a standard component in many courses. E-learning is recognised as a strategically important element for the provision of learning across all areas of society. However, some important issues arise [1]:

- Institutions must provide an adequate and reliable technical infrastructure to support elearning activities.

- Instructors and learners must process the technical skills to use e-learning tools.

- Instructors must redesign their courses to incorporate e-learning effectively into their pedagogy.

\author{
Faiçal Azouaou \\ Higher School of Computer Sc. (ESI)
}

- Both Instructors and learners need to collaborate with their peers in order to share their knowledge and experiences.

We are interested in our research to the last issue and we try to provide learners and instructors with a technology support in order to facilitate their exchange and sharing of knowledge resources and experiences. We consider that both learners and instructors collaborate through online communities.

One of the most important areas within group collaboration is the current research on communities of practice (CoPs) [2] where people share common professional objectives and whose collaborative relationships support the organizational goals.

Online CoPs need virtual frameworks that enable the creation of knowledge, and facilitate the exchange between members. Wikis are considered as suitable tools for creating and sharing knowledge. Using wikis in CoPs, allow members to create pages, share knowledge resources and work collaboratively. However, as the amount of knowledge produced grows rapidly, one of the most important issues of CoPs is the management of the produced knowledge (i.e. structuring and representing, searching and reusing...). Accordingly, we are focusing in our research works to support members of CoPs by tools and services based on the semantic web technologies. Using semantic web technologies will help them to manage their knowledge efficiently.

In this paper we focus on the design and implementation of a semantic wiki for CoPs of teachers from the higher education context. A semantic wiki is a wiki that offers the flexibility of wikis, an intuitive interface, together with knowledge management features [3]. We aim to favor the knowledge sharing within the $\mathrm{CoP}$, using the semantic wiki. This tool called EasyLearningWiki, is considered as a support for teachers who want to collaborate and exchange knowledge, tacit and explicit, to build parts of their courses online for their students [4].

The wiki is developed using an ontology, exploited first to describe the knowledge resources added to the wiki by means of objective annotations, and then to allow users (i.e. members of the $\mathrm{CoP}$ ) to express their feedback and assessment regarding these same resources through subjective annotations. In addition, EasyLearningWiki provides a personalized access to the wiki according to the 
member's profile (i.e. adapting the interface and functionalities).

The rest of the paper is structured as following: Section 2 expresses the objectives and needs of online CoPs of teachers. Section 3 presents the main concepts of semantic wikis and some related works. The next sections describe the EasyLearningWiki, its objective, main functionalities, as well as the semantic and technical aspects. Finally, the conclusion highlights the main results and presents some future perspectives.

\section{Online Communities of practice}

\subsection{Online CoPs of teachers}

According to Wenger [5], CoPs are "groups of people who share a concern, a set of problems, or a passion about a topic, and who deepen their knowledge and expertise in this area by interacting on an ongoing basis". By using information and communication technologies, online CoPs bring members together virtually, to learn from each other, collaborate and share expertise and knowledge.

We consider CoPs made up of members from the higher education context (faculties, lecturers, teaching assistants, lab assistants). Those actors have different levels of skills and experience. They need to have the possibility to collaborate and exchange their knowledge, tacit and explicit, related both to pedagogical and domain expertise. Members need especially to find the adequate resources according to their needs and interests, and also to be noticed on recent updates and news. They aim to collaborate, exchange efficiently by reusing their tacit and explicit knowledge (e.g. e, reuse a complete/partial answer or some hints/examples).

\subsection{CoP's needs}

The authors in [6] specify and categorize CoPs' needs into four categories: (1) support participation; (2) constitute common resources; (3) support commitment; and (4) support realization of the activities. This categorization is based on the needs expressed by the CoPs involved in the PALETTE project [7]. A set of services is proposed in this project including: (1) knowledge management services, for knowledge creation and enrichment, knowledge retrieval, knowledge dissemination, knowledge visualization, knowledge evaluation, and knowledge evolution services; (2) mediation services, supporting argumentative and collaborative design and decision making; and (3) information services, for information production and reuse.

We focus in this research on the second need of CoPs, constitute common resources, which means facilitate the knowledge exchanges and sharing between members through the capitalization of tacit and explicit knowledge. Accordingly, it is necessary to formalize tacit knowledge; archive common resources and make them retrievable and reusable.

In the next sections, we present EasyLearningWiki, a semantic adaptive wiki we propose for members of our community in order to manage their knowledge more efficiently. Different kinds of knowledge resources can be distinguished: learning resources, learning scenarios, problems /solutions, best practices, etc. The tool is suitable for the community as it offers some knowledge management features, including structuring and representing knowledge, searching and tagging supports, as well as the notification and the personalized access according to the member's profile.

\section{Semantic wikis}

In the beginning [8], internet websites and tools were simple and had basic functionalities. The web pages were written in html and were considered as a simple text augmented by links to other pages, these links are called hypertext.

But since, its creation, the internet has evolved into three directions:

- Interactive. There are now tools that enable internet users to create and publish their own content on the web. These tools can be: blogs, forums, and video sharing tools...internet users are becoming less passive and more and more active. Wikis are one of these tools that permit users to create documents and use them in a collective manner.

- Social. With the emergence of social networks, such as Facebook and MySpace, Internet users can now create social connections with friends from around the world, and can share with interesting content.

- Semantic. Using semantic web technologies, Internet resources, such as web documents, will have an explicit semantics that can be processed by semantic engines or other software agents. This semantics will enable more sophisticated processing, as the web would look like a large database or knowledge base.

Wikis are interactive tools used to build social web sites enabling a large number of users to create a new page or modify any page using their web browser. However, as they grow, wikis suffer from a number of problems such as anarchical structure, large number of pages, aging navigation paths [9].

A semantic wiki is a wiki engine that uses semantic Web technologies to embed formalized semantics/knowledge, content, structures and links, in the wiki pages. Therefore, this knowledge can be used to propose enhanced features such as better 
knowledge resources searching, suggesting new links, identifying linked networks, dynamic content update, checking and notification, etc.

The state of the art shows the development of many semantic wiki projects. Buffa et al. [10] distinguish between two approaches: those considering "the use of wikis for ontologies" and those considering "the use of ontologies for wikis".

From the first category: the wiki Platypus [11] supports basic ontology editing but with no consistency check between the annotations and the ontology. It does not come with a reasoning engine and supports only basic queries. Semantic metadata are used for improving navigation but the main drawback is that the users have to switch between editing normal text and editing semantic annotations as these activities are done using two distinct textbased editors.

For the second category, we present: SweetWiki $[3,10]$, developed with semantic web technologies that is based on the concept of "social tagging" (or social annotation). Using a WYSIWYG editor, the wiki's user can tag (allocate keywords to) pages, pictures, attached files, etc. The semantic research module CORESE is automatically and transparently used on these tags. If a user types a tag in the research form, he will receive all the objects tagged with this tag or its sub-tags. The user will then receive all the related tags that are linked with relevant documents. SweetWiki also offers an editor of "folksonomy" (i.e. a set of interrelated tags). Then, each user can organize the tags.

\section{EasyLearningWiki: Objectives and functionalities}

This section describes the objectives and functionalities of EasyLearningWiki, dedicated for CoPs of teachers from the higher education context.

\subsection{Objectives}

EasyLearningWiki aims to provide members of the CoP with a virtual space for sharing their knowledge resources, allowing: (1) a collaborative production and edition of resources between the different members (faculties, lecturers, teaching assistants, lab assistants); (2) facilitating access to knowledge resources; and (3) providing a personalized access. The objective is to increase the degree of reuse and exploitation of tacit and explicit knowledge of the CoP, increase the active participation rate, and enhance the individual and organizational learning.

Adding a new resource implies the objective annotation of this resource (i.e. a structured description based on the use of an ontology). Furthermore, EasyLearningWiki allows a subjective annotation on the resource based on the member's annotation model to capture the member's feedback about the use of this resource.

The adaptation of the wiki according to the member's profile occurs on three levels:

- Information search and retrieval: from the initial request of a member, we can add information, constraints, conditions to generate a new query whose result is adapted to the member's profile.

- Notification: each member is notified with personnalized information according to his profile (e.g. for a new resource, an update or an evaluation).

- Adaptative interface: the wiki interface is personalized and adapted according to the member's information profile. For example: editing resources that are recently added, viewed or updated or those annotated with subjective annotations either by the member or by members with similar profile. The wiki offers also a dynamic exploitation of the member's information profile taking into account both the explicit information provided by the member itself and the implicit information captured from his different interactions such as his preferences, needs, relations with other members, etc.

\subsection{Functionalities}

EasyLearningWiki offers the functionalities of a wiki together with some knowledge management features, such as:

- Tagging support: the member is provided with a toolbar to add tags, to tag a page or parts of it (e.g. included knowledge resources). Tagging has two benefits: (1) a better knowledge resources searching, enables to find easily the tagged resource when searching for it, and (2) a navigation support, enables to have access to other resources tagged with concepts related to the one(s) used to tag the resource.

- Semantic search engine, using SPARQL queries allow members to find easily resources as they are described semantically with objective and subjective annotations or tagged with a set of tags.

Notification: members are provided with semantic awareness information. They can be notified on recent modifications on pages corresponding to a set of tags they have already chosen. They can be also notified on knowledge resources corresponding to a set of tags they have already chosen and evaluated with high scores by members having the same profile and/or having a high level of expertise in the domain of interest. 
- Folksonomy edition: tags are linked together within a folksonomy in order to organize them. The folksonomy is described using the semantic web languages, where tags are related to one another using relationships like subClassOf. In addition, it is possible to merge concepts when two tags are synonymous for example.

\section{EasyLearningWiki: Semantics}

In this section we present the semantic aspect of EasyLearningWiki. Different ontologies are considered: an application domain ontology, covering the knowledge of a specific course (e.g. software engineering, networking...), a domain ontology describing the main concepts of a CoP, including the "Profile" and the "Knowledge resources", which will be presented in the next subsections.

\subsection{Semantic annotations}

The main goal of the Semantic Web is to offer complete solutions for the content of Web resources by adding semantics in the form of metadata or annotations to make them machine-interpretable [8]. This type of annotation is called "semantic annotation" and is defined as "a formal representation of content, expressed using concepts, relations and instances described by means of an ontology, and linked to the resource". It is based on an existing knowledge model (ontology), linking the entities of the resource and their semantic descriptions represented in the ontology [12].

Several semantic annotation models are proposed. In pedagogical context, Marshall [13] studied the characteristics of annotations among students. Mille [14] presented the learners' annotations on pedagogic documents and considered the annotation as a set of attributes organized in distinct but complementary categories. Azouaou [15] proposed the annotation model for a personal memory of the teacher. In addition, we mention some other works for example in the context of semantic web [16] and for electronic documents [17]. Many frameworks have been proposed such as: Annotea [18] and SCREAM [19].

Our objective is to describe the CoP's resources using semantic annotations. We consider two types of semantic annotations: (1) objective annotations, describing the knowledge resources with a set of metadata, and (2) subjective annotations, to express the members' feedback regarding these same resources.

Figure1 below illustrates an excerpt of the conceptual model of the CoP's domain ontology. Different types of resources can be distinguished: learning object, learning scenario, problem/solution, best practice, etc. Each resource is described using semantic annotations and each member is characterized with his own profile.

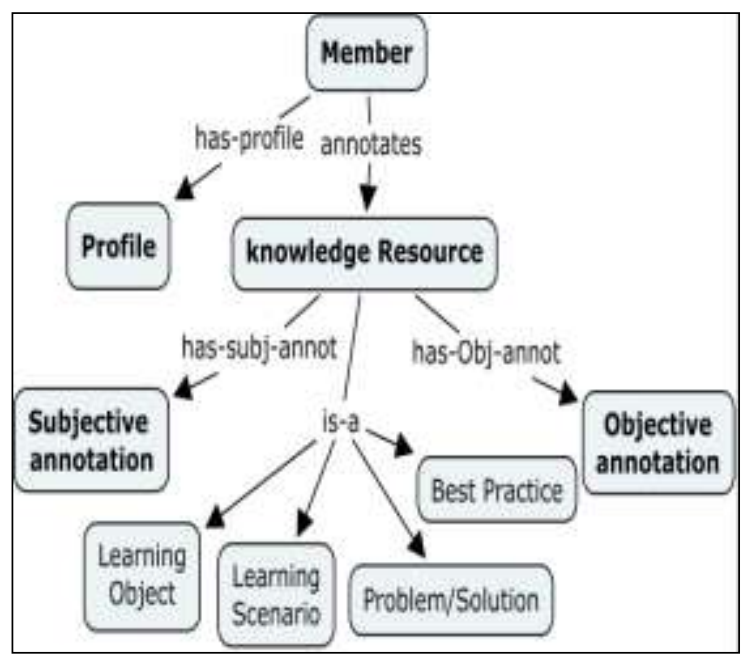

Figure 1. Excerpt of the conceptual model of the CoP's domain ontology

We present below the two types of semantic annotations, objective and subjective, as follows:

1) Objective Annotation: represents a set of metadata that describe the resource. We consider the following levels inspired from the model proposed by Azouaou [15] :

- The Cognitive level: represents the physical anchor and the visual form of the annotation as proposed by Azouaou [15]. At this level, the member highlights some important parts of the resource.

- The contextual level: represents the context in which the member annotates the resource before to add it to the wiki (e.g. the utilization context of the resource)

- The semantic level: represents the semantics of the annotated resource by linking it to some concept from the knowledge model ontology.

2) Subjective annotation: we consider the following levels :

- The Cognitive level: represents the physical anchor and the visual form of the annotation as proposed by Azouaou [15].

- The feedback level: members can express their feedback through comments and evaluations. We provide a set of metadata allowing members to evaluate a learning resource (e.g. the difficulty, score) as well as a means of expressing an opinion on the resource through 
comments. The evaluations and comments can be exploited by other members of the wiki.

\subsection{Member's profile}

A generic member's profile model for CoPs of elearning is presented in [20]. The model is based on two types of information: static and dynamic information (see Figure. 2):

- Static information: includes personal characteristics of the member such as contact details, academic background, working experience, member status (e.g. expert, novice), qualification (e.g. technical, pedagogical), specialty, languages, etc.

- Dynamic information: based on five elements: Preferences and Needs regarding the use of knowledge resources, as well as Relations among individuals. Experience reflects members' expertise and know-how about a specific domain. Competences refer to cognitive characteristics such as the creativity, perception and social skills.

We have adopted this model in our study.

Figure 2 represents a screenshot of the ontology developed using Protégé. The figure illustrates the semantic annotation of learning resource and member's profile. To annotate a learning resource we use the Learning Object Metadata standard (LOM) [21] to ensure the interoperability between our wiki and other online learning systems (i.e. when importing a learning resource from another system). Furthermore, we propose other metadata such as the author, date and context of use (e.g. learning domain, learning level...). Furthermore, the member can use some visual forms in order to highlight the importance of some important information of the resource. However, in order to facilitate the capture and to encourage the members to be active and to share their knowledge resources, we chose to not require the capture of all metadata (i.e. some of them will be optional).

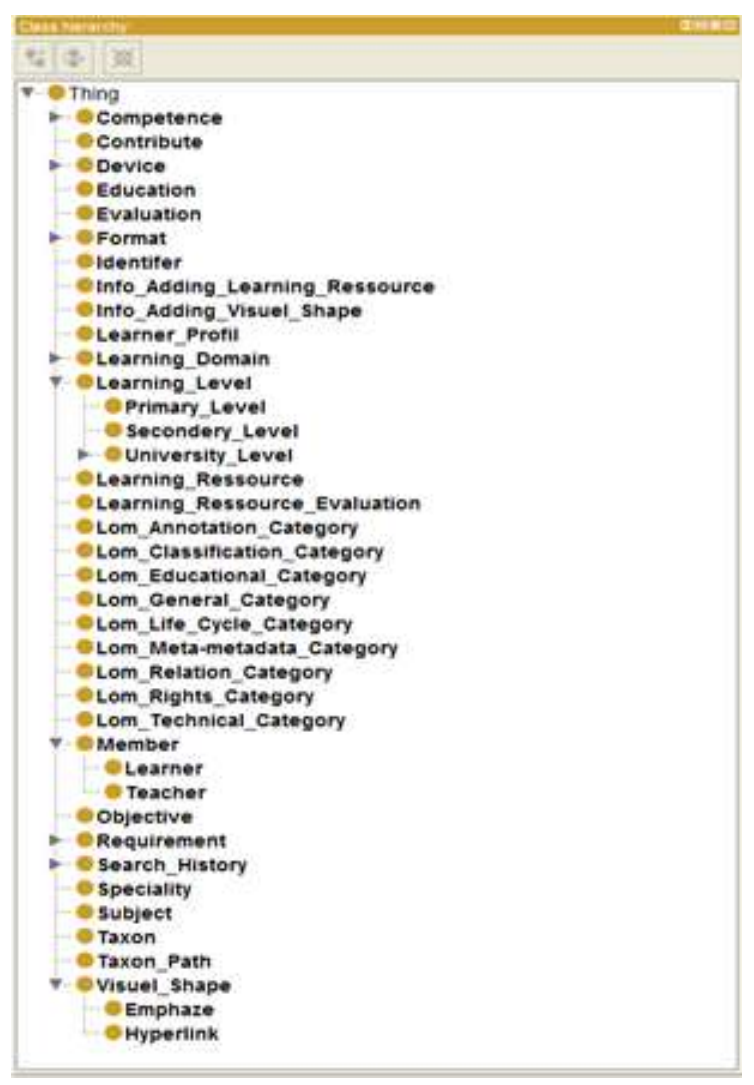

Figure 2. The ontology representation using Protégé editor

\section{EasyLearningWiki: Technical Architecture}

EasyLearningWiki is based on different components: ontologies, semantic annotations and tags, resources, and a set of modules:

- Semantic search engine: based on SPARQL queries [22] and also on similarity metrics;

Browsing: using queries and navigation tags,

- WYSIWYG editor: is used for content, annotations and tags editing,

- Tagging support: for the management of the tags.

- Notification: based on the semantic annotations and tags and using the member's profile information, the module will notify the member by the last updated resources containing the tags which interest him; or by the new resources added to the wiki and that are interested for him, etc.

- Annotation: for objective annotations (add/update resources), deleting resources, or annotating subjectively resources.

Figure 3 illustrates the architecture of the semantic wiki structured into three layers: Interface layer, semantic and data layer and application layer. 


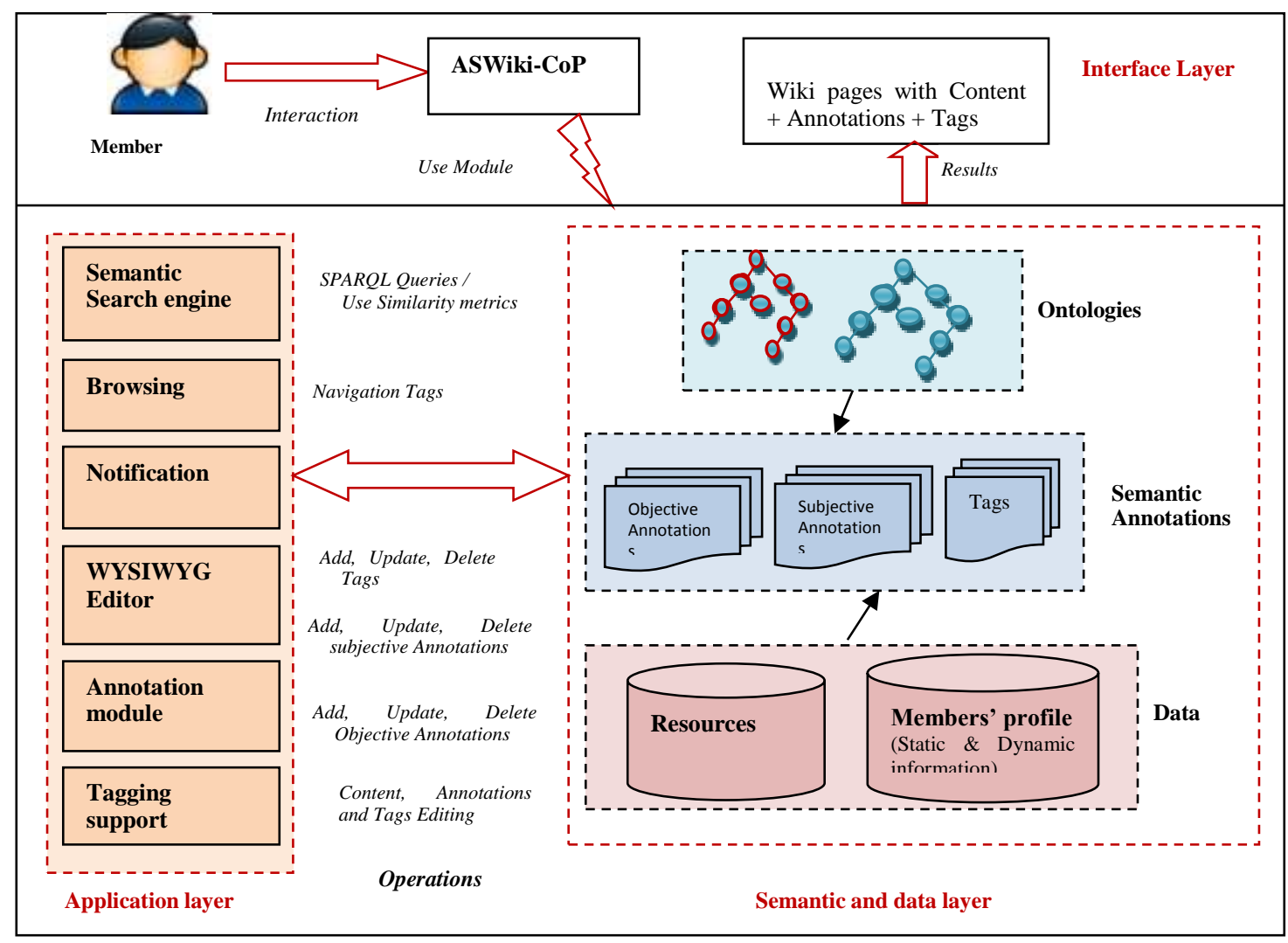

Figure 3. EasyLearningWiki architecture.

The member (i.e. learner/teacher) has first to login to EasyLearningWiki (see figure 4).

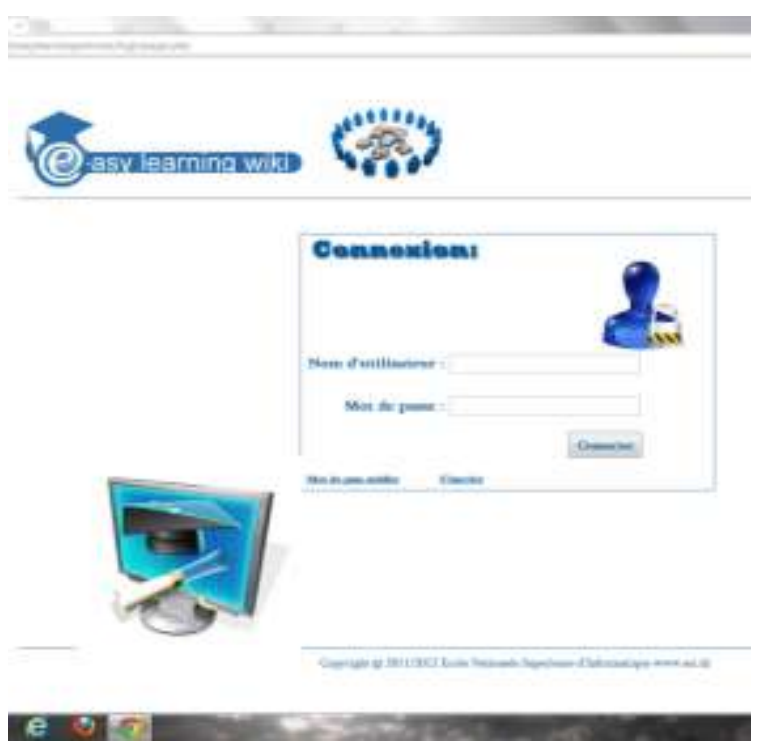

Figure 4. A screenshot of the EasyLearningWiki homepage.

The member opens an existing document, upload an offline document, or event create a new one from scratch online using a rich editor (see figure 5).
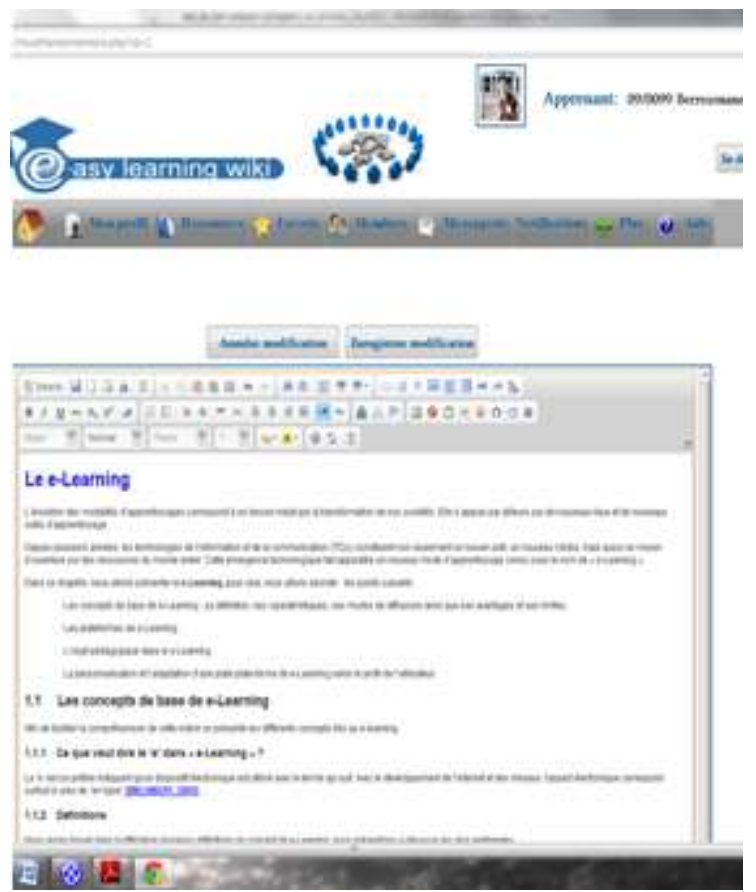

Figure 5. A newly created document using the online rich editor.

Each time a new document is added to EasyLearningWiki, the member has to describe it semantically suing values from the ontologies (see figure 6). 


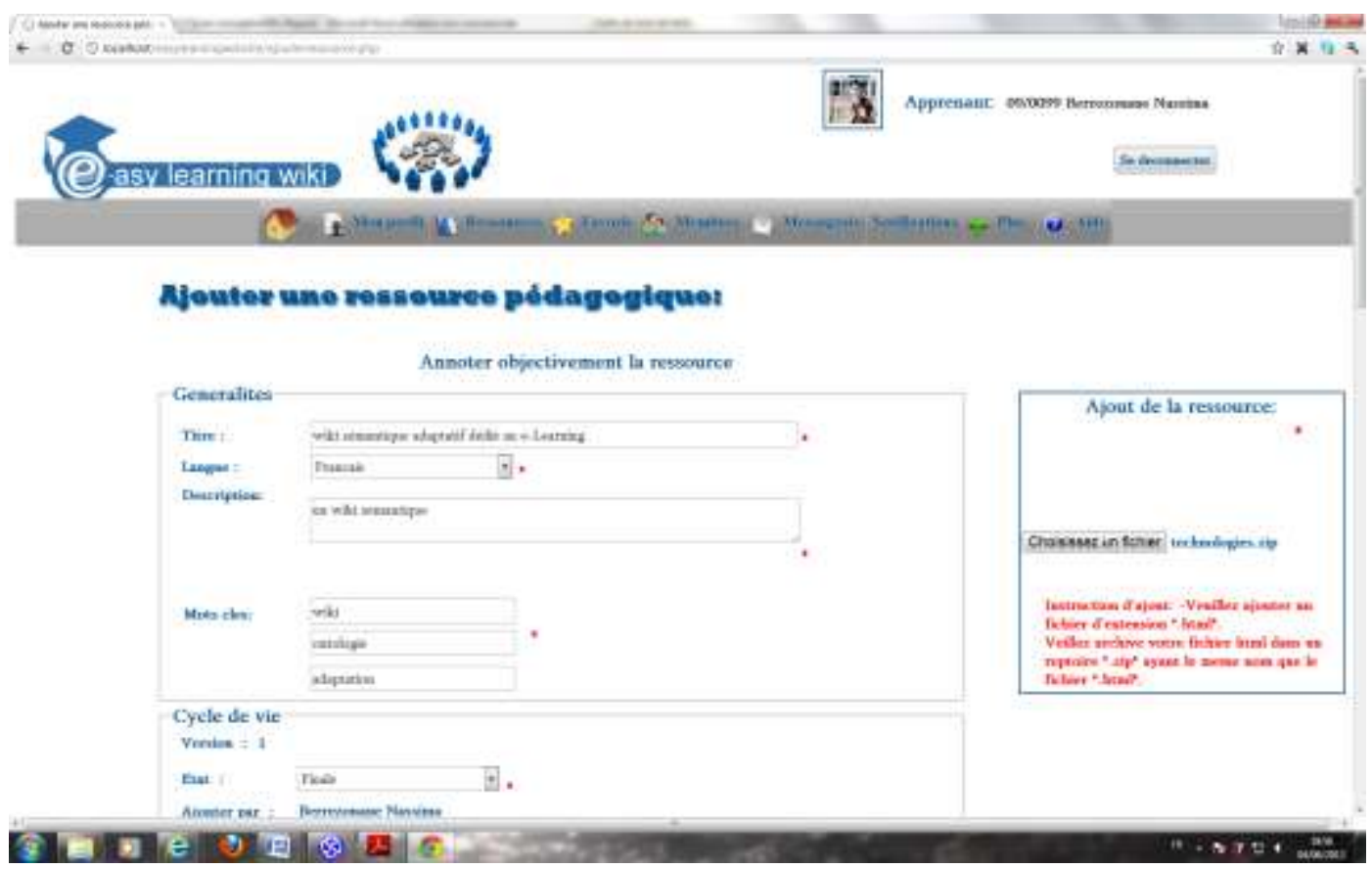

Figure 6. Describing a new added ressource (objective annotation).

Every member can annotate the wiki documents in an objective manner (see figure 6) or a subjective one (see figure 7).

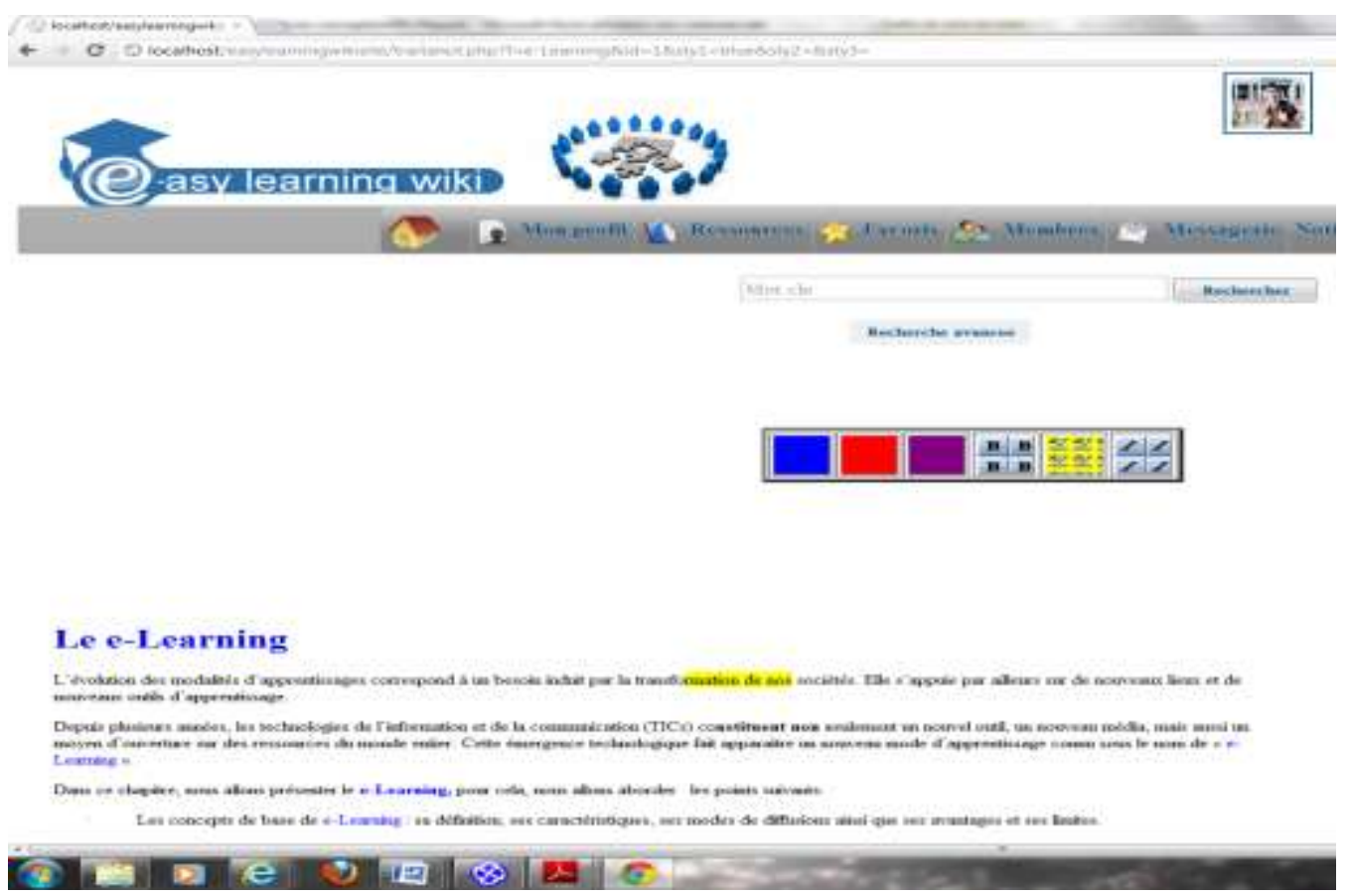

Example of a subjective annotation.

\section{Conclusion}

Semantic technologies are promising to significantly enhance learning and teaching in higher education. The emergence of Web 2.0 strategies for enabling learning content annotation and the adoption of semantic web have introduced a new and promising perspective, which has been explored in the context of higher education [23]. 
This paper presents an adaptive semantic wiki dedicated for CoPs of teachers in the higher education context. Our objective is to support members of those CoPs to collaborate and exchange efficiently providing them with a set of services for sharing and reusing their knowledge, tacit and explicit.

The presented wiki is based on the semantic web technologies, using semantic annotations for the capitalization of knowledge. The paper proposes a member's annotation model based on two types of annotations: (1) objective annotations to describe the knowledge resources; and (2) subjective annotations to express the member's feedback regarding these same resources. Furthermore, the wiki provide an adaptive access according to the member's profile.

In our future work, we envisage to complete the development of the different components of the wiki: the semantic search engine, the tagging support, and the notification service taking into account the dynamic aspect of the member's profile.

\section{References}

[1] A., Judith, "Supporting E-learning in Higher Education". Pirani, Research Fellow, Educause Center for Applied Research.

[2] E., Wenger, "Communities of Practice: Learning as a Social System. Systems Thinker”, 1998.

[3] A., El Ghali, A., Tifous, M., Buffa, A., Giboin, R., Dieng-Kuntz, "Using a Semantic Wiki in Communities of Practice", in Proceedings of the 2nd International Workshop on Building Technology Enhanced Learning. Solutions for CoPs, TEL-CoPs'07, EC-TEL'07, Crete Greece, 18 September, 2007, pp. 22--31, 2007.

[4] L., Berkani, F., Azouaou, "An adaptive semantic wiki for CoPs of teachers-Case of higher education context". Proceedings of the International Conference on Information Society (i-Society), 2012, pp. 472-477.

[5] E., Wenger, R., McDermott, W.M., Snyder, "Cultivating Communities of Practice: A guide in Managing Knowledge”. Harvard Business School Press, 2002.

[6] B., Charlier1, A., Boukottaya, A., Daele, N., Deschryver, S., El Helou, Y., Naudet, "Designing services for CoPs : first results of the PALETTE project", in Proceedings of the 2nd International Workshop on Building Technology Enhanced Learning Solutions for CoPs, TEL-CoPs'07, EC-TEL'07, Crete Greece, 18 September, 2007.

[7] PALETTE IST project, "Pedagogically sustained Adaptive Learning through the Exploitation of Tacit and Explicit Knowledge", 2006, http://www.ecrim.palette.org.

[8] T., Berners-Lee, J., Hendler, O., Lassila, "The Semantic Web". Scientific American, pp. 29--37, 2001.
[9] M., Buffa, F., Gandon, F, "SweetWiki : Semantic WEb Enabled Technologies in Wiki", in WikiSym, IEEE International Symposium on Wikis, August 21-23, Odense, Denmark, 2006.

[10] M., Buffa, F., Gandon, G., Ereteo, P., Sander, C., Faron, C., "SweetWiki: A semantic wiki, Special Issue of the Journal of Web Semantics on Semantic Web and Web 2.0”, Vol. 6, Issue 1, Elsevier, pp. 84-97, 2008.

[11] S.E. Campanini, P. Castagna, R. Tazzoli, "Platypus Wiki: a Semantic Wiki Web", in Proceedings of the 1st Italian Semantic Web Workshop, Ancona, Italy, 2004

[12] F., Amardeilh, "Semantic Web and Linguistic Computing: methodological proposals and implementation of a software platform". PhD Thesis, Paris X University, 2007.

[13] C., Marshall, "The future of annotation in a digital (paper) world", in 35th Annual GSLIS Clinic: Successes and Failures of Digital Libraries, University of Illinois at Urbana-Champ, 2000.

[14] D., Mille, " Models and Software Tools for Semantic Annotation of Learning Documents", PhD Thesis, Computer Science Departement, Joseph-Fourier University, Grenoble, p. 173 pages, .2005 .

[15] F., Azouaou, "Models and Annotation Tools for a Personal Memory of the Teacher". PhD thesis computer science, Joseph Fourrier University, Grenoble, 2006

[16] J., Euzenat, "Eight Questions about Semantic Web Annotations". IEEE IntelligentSystems, 17(2), pp. 55-62, 2002.

[17] M.Veron, "Modeling Annotative Component in Electronic Documents". Master Thesis, IRIT University, Toulouse, 1997.

[18] J., Kahan "Annotea: an open RDF infrastructure for shared Web annotations". Computer Networks, 39(5): pp. 589-608, 2002.

[19] S., Handschuh, S., Staab, F., Ciravegna, "SCREAM - Semi-automatic CREAtion of Metadata", in Gòmez-Pérez, A., Benjamins, V.R. (Eds.):EKAW, LNAI 2473, pp. 358-372, 2002.

[20] L., Berkani, A., Chikh, "A process for knowledge reuse in communities of practice of e-learning". Procedia Social and Behavioral Sciences, Innovation and Creativity in Education, vol. 2, no. 2 pp. 4436-4443, 2010.

[21] LOM, Learning Object Metadata, http://ltsc.ieee.org/wg12/

[22] SPARQL Query Language for RDF http://www.w3.org/TR/rdf-sparql-query/

[23] Tiropanis, T., Millard, D., Davis, H.C. (2012). Guest Editorial: Special Section on Semantic Technologies for Learning and Teaching Support in Higher Education. IEEE Published by the IEEE Computer Society, Vol. 5, No. 2, pp. 102-103. 